\title{
Comparative evaluation of pulpal vitality test accuracy in different groups of teeth
}

\author{
Elnaz Mousavi ${ }^{1 *}$, Narges Simdar ${ }^{1}$, Sara Mehri ${ }^{2}$, Maryam Ghamari ${ }^{3}$ and Sepideh Arab ${ }^{4}$ \\ ${ }^{1}$ Assistant Professor, Department of Endodontics, Faculty of Dentistry, Guilan University of Medical Science, \\ Rasht, Iran \\ ${ }^{2}$ Dentist \\ ${ }^{3}$ Assistant Professor, Department of Endodontics, Faculty of Dentistry, Ghazvin University of Medical \\ Science, Ghazvin, Iran \\ ${ }^{4}$ Assistant Professor, Department of Orthodontic, Faculty of Dentistry, Tehran University of Medical Science, \\ Tehran, Iran
}

\begin{abstract}
The vitality pulp tests are necessary for diagnosis of pulpal disease and differentiation between endodontic and nonendodontic disease. Thermal and electrical tests are conventional method for evaluation pulp vitality. The aim of the present study was to evaluate the accuracy of thermal and electrical tests to register pulp vitality in different group of teeth. 184 teeth (81 male, 103 female) with unknown pulpal status that need endodontic treatment were examined. After complete isolation, the thermal and electrical tests were performed. The cold, heat and electrical tests were done. The interval between tests was conducted after 2 minutes. Based on information obtained the sensitivity, specificity, accuracy, positive and negative predictive value were calculated. In total, 78 teeth with necrotic pulp and 106 teeth with vital pulp were tested. The gold standard was established by direct pulp inspection of 184 teeth with endodontic treatment. Based on information obtained the accuracy of cold test in anterior teeth was $78 / 8 \%$, in premolar teeth was $81.8 \%$ and in molar teeth was $80.5 \%$. The accuracy of heat test in anterior teeth was $82.6 \%$, in premolar teeth was 78.1\% and in molar teeth was 72.7\%. The accuracy of electric pulp test in anterior teeth was 71.1\%, in premolar teeth was $74.5 \%$ and in molar teeth was71.4\%. In anterior teeth the heat test has more accuracy than the other one. In premolar and molar teeth the cold test has more accuracy than other test.
\end{abstract}

KEY WORDS: DENTAL PULP TESTS, DIAGNOSIS

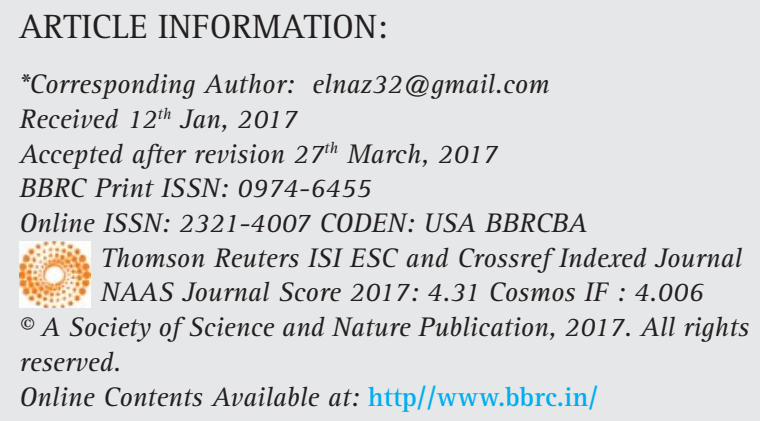


INTRODUCTION

Diagnosis in dentistry may be defined as the process whereby the data obtained from questioning, examining and testing are combined by the dentist to identify deviations from the normal. The diagnosis of dental pulp status should be seen as a synthesis of history, clinical examination, special tests, and radiological examination, and not as the outcome of any one specific test. Vitality testing is an important aid in the diagnosis of pulp disease and apical periodontitis (Gopikrishna et al. 2009). The complex anatomical structure and inaccessibility of the dental pulp to clinical tests makes an accurate diagnosis of the health of the dental pulp often difficult and challenging to the dentist. The physical confunement of the dental pulp, with its rich sensory nerve innervations and microcirculatory components make the dental pulp a unique tissue (Samuel et al. 2014).

Conventional pulp tests such as electric pulp tests and thermal tests measure the neural component of the pulp and are now considered as pulp sensibility tests (Lin et al. 2008). Studies have shown that blood circulation, and not the nerve innervation, is the accurate determinant of the pulp vitality as it provides an objective differentiation between vital and non-vital pulp (Yu and Abbott, 2007). Hence, pulpal circulation is the true determinant of pulp vitality. The nerve fibers of the pulp may be resistant to necrosis than the vascular tissue and may be reactive long after the surrounding tissues have degenerated (Gopikrishna et al. 2007).The conventional tests are subjective and depend upon the patient's perceived response to the stimulus as well as the dentist's interpretation of that response. Pulp sensibility tests have limitations, and false responses can occur (Bender, 2000). Electric pulp tests are known to be unreliable in immature teeth and in teeth undergoing orthodontic movement (Myers, 1998). Furthermore, false responses are known to occur when the electric current is conducted to adjacent periodontal tissues, adjacent teeth and even to remnant inflamed pulp tissue with liquefactive pulp necrosis. In traumatized teeth, Electric pulp tests are less reliable than cold tests It is also reported pulpal sensibility testing with Endo Ice and pulp tester are more accurate and reliable methods of determining pulpal vitality, (Chen et al. 2011 and Jespersen et al. 2014).

Cold tests are known to elicit false-negative responses in elderly patients because of the amount of thermal insulation provided by secondary dentin (Emshoff et al. 2004). Commonly available agents for cold tests are $\mathrm{CO}_{2}$ snow, ice, and refrigerant sprays (eg, tetrafluoroethane, butane, propane, isobutane, dicholorofluoromethane and ethyl chloride). Cold tests and electric pulp tests are pulp sensibility tests that have been established as useful aids in the assessment of pulp status despite being subjective and patient-dependent (Emshoff et al. 2004). Nonetheless, the lack of pulp sensibility is often associated with advanced pulp necrosis (Chen et al. 2011).

Currently, there is no reliable and acceptable pulp testing method for the pediatric age-group. The aim of the current study was to determine sensitivity and specificity of cold, heat and electric test on pulpal vitality tests in different dental type.

\section{MATERIAL AND METHODS}

\section{PATIENTS}

A total 184 teeth (81 male, 103 female) with unknown pulpal referred to Endodontic Department of dental clinic of international branch of Guilan University of Medical Sciences during 2015 were examined. Patients aged 12-81 (mean 37.94 years old). They informed about the study and signed the form. The inclusion criteria were not suffering systemic disease or administration of NSAID drugs.

\section{STUDY PROTOCOL}

Then peri-apical radiography was done on teeth and those with calcification and decay without pulp invasion were included into as case group. The next tooth in the same arc with no calcification or decay kept as control. At first, primary diagnosis was done in all pulp based on observation followed by observation. After complete isolation, the thermal and electrical tests were performed in case and control teeth. The cold test by ethyl choloride and heat test by gutta percha and electrical test with electrical pulp tester (Parkel/USA) were done. Two minutes interval between tests was conducted. The pain response of the patients was recorded as normal, high and highest $(+,++$ and +++$)$. Based on information obtained the sensitivity, specificity, accuracy, positive and negative predictive value were calculated for each method in different groups of teeth.

\section{STATISTICAL ANALYSIS}

The obtained data was analyzed using SPSS ver. 21 using One Way ANOVA. For treatments showing a main effect by ANOVA, means were compared using chi squar test. $\mathrm{P}<0.05$ was considered as significant differences between treatments.

\section{RESULTS}

The results of the accuracy of thermal and electrical tests to register pulp vitality in different group of teeth are presented in figures 1-14. A total 184 teeth (81 male, 103 female) with unknown pulpal status that need endo- 

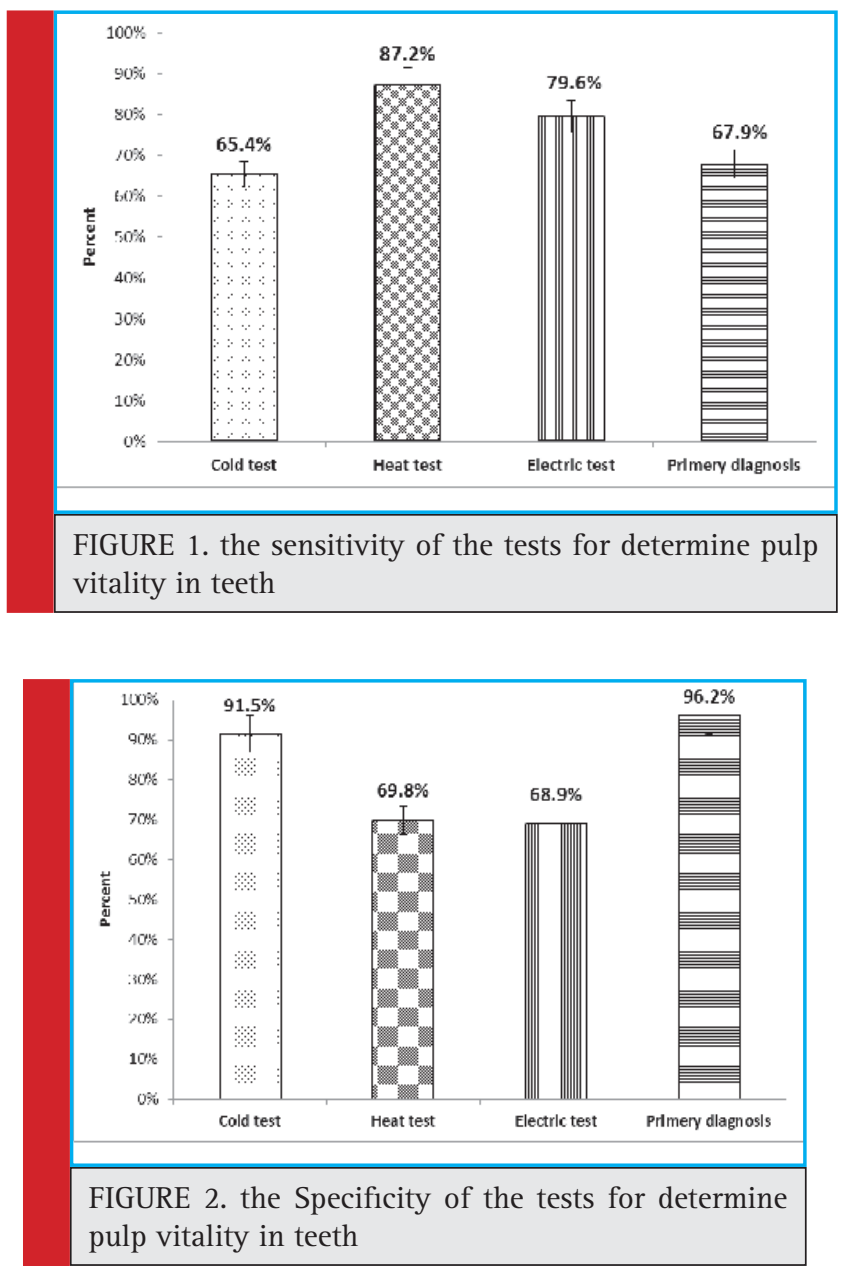

dontic treatment were examined. In total, 78 teeth with necrotic pulp and 106 teeth with vital pulp were tested. As seen in figure 1, the heat test had the highest sensitivity (87.2\%) compared to the other tests for determine pulp vitality in teeth.

As observed in figure 2, primary test (96.2\%) and cold test (91.5\%) had the highest accuracy of the tests for determine pulp compared to the other tests, respectively.

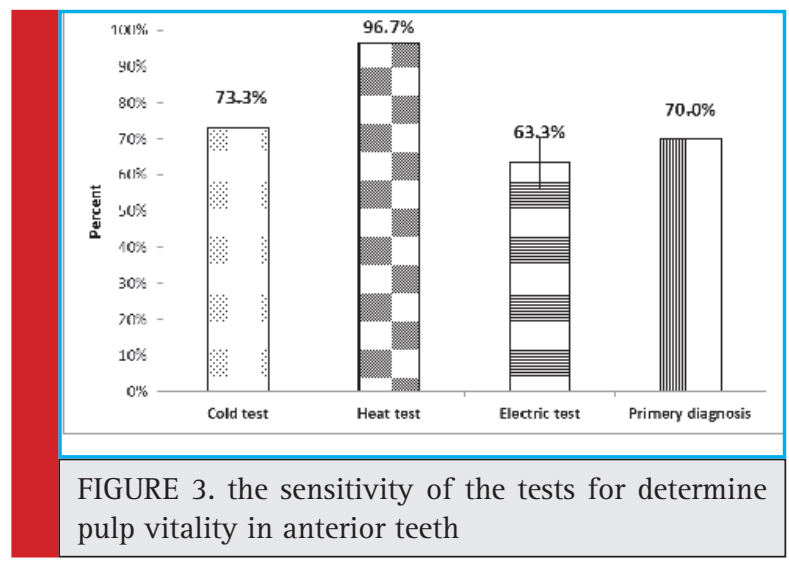

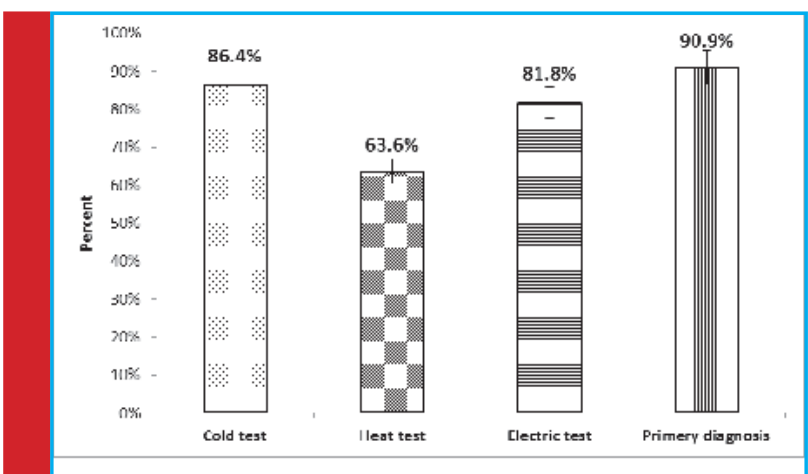

FIGURE 4. the Specificity of the tests for determine pulp vitality in anterior teeth

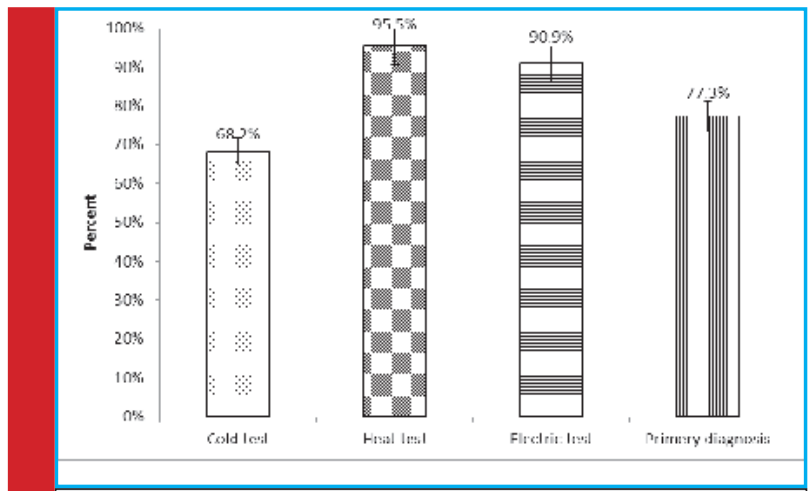

FIGURE 5. the sensitivity of the tests for determine pulp vitality in pre-molar teeth

Among the various tests, the heat test had highest sensitivity $(96.7 \%)$ for determine pulp vitality in anterior teeth (figure 3).

Furthermore, the heat test had lowest Specificity $(63.3 \%)$ of the tests for determine pulp vitality in anterior teeth (figure 4).

The results of the sensitivity of the tests for determine pulp vitality in pre-molar teeth is presented in figure 5 . As seen, heat test (95.5\%) and electric test (90.9\%) had

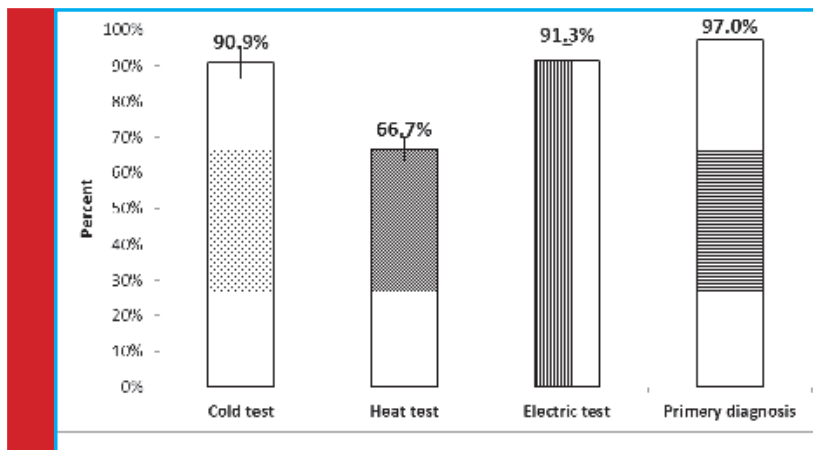

FIGURE 6. the Specificity of the tests for determine pulp vitality in pre-molar teeth 


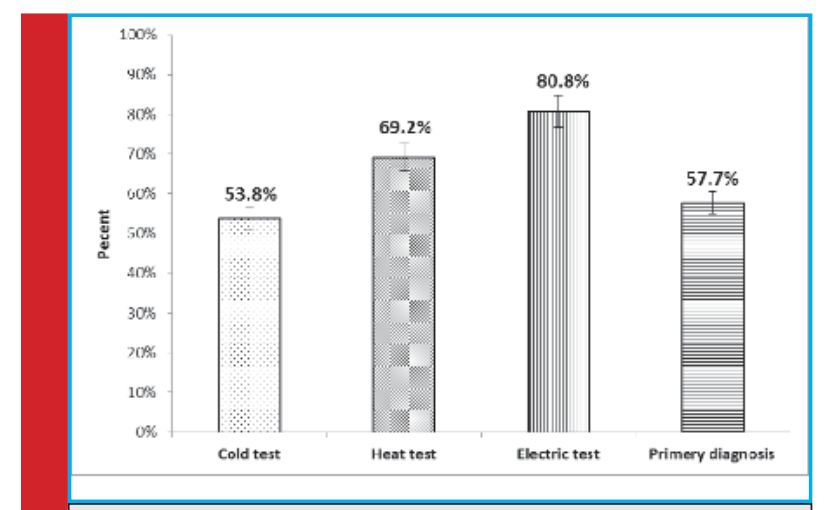

FIGURE 7. the sensitivity of the tests for determine pulp vitality in molar teeth

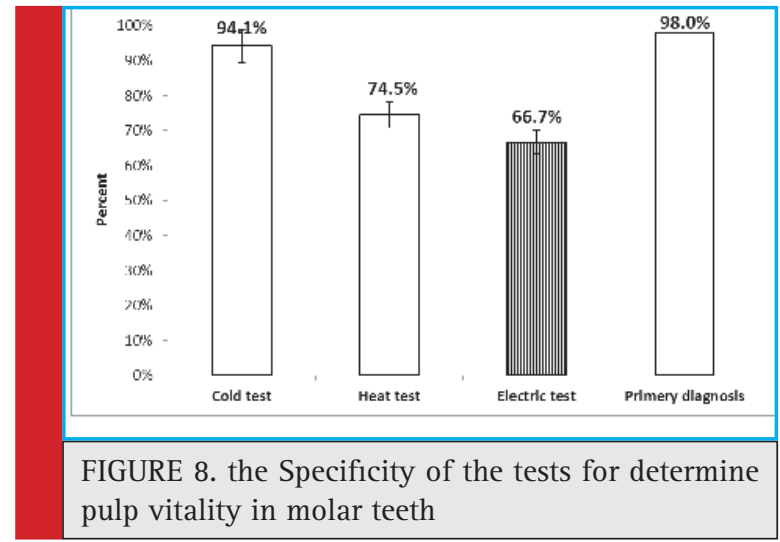

the highest sensitivity of the tests for determine pulp vitality in pre-molar teeth, respectively.

Also, the heat test had lowest Specificity (66.7\%) for determine pulp vitality in pre-molar teeth (figure 6).

As seen in figure 7, for the sensitivity of the tests for determine pulp vitality in molar teeth, the electric test was highest (80.8\%) compared to the other tests.

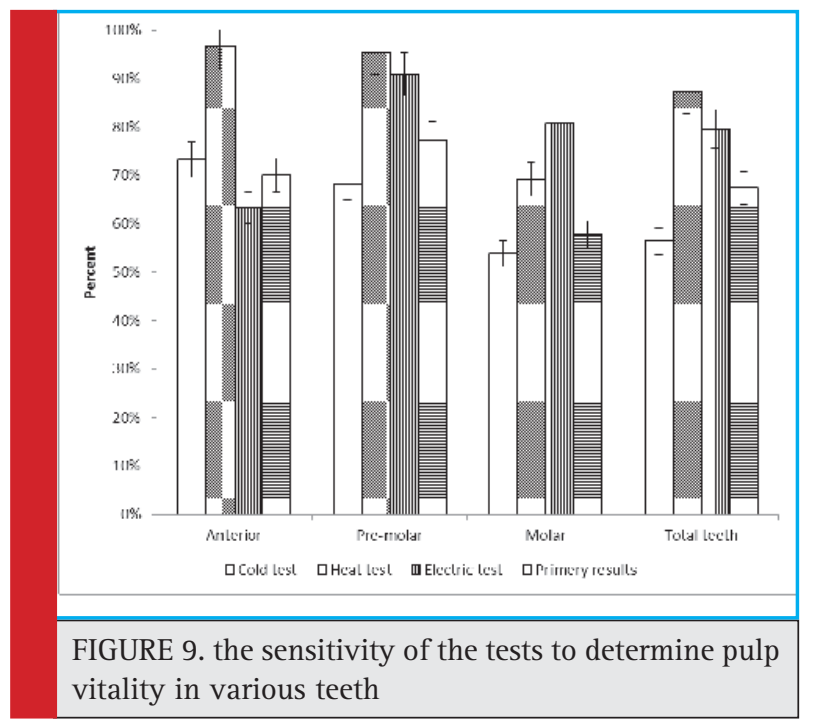

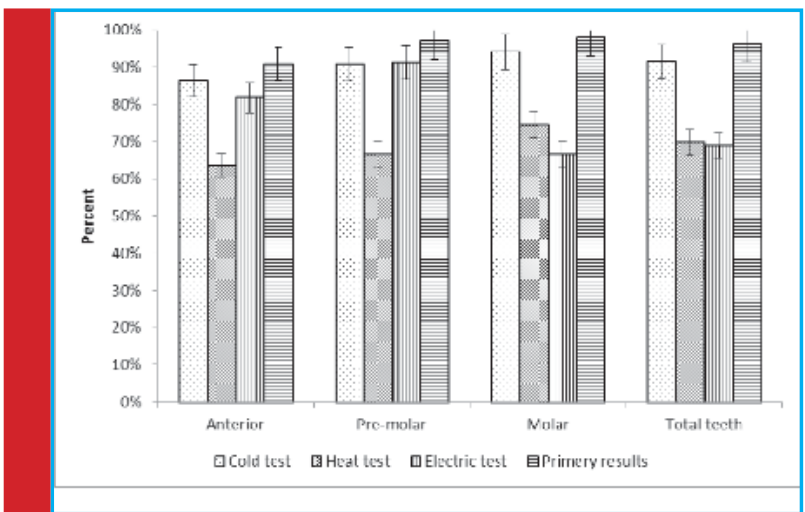

FIGURE 10. the Specificity of the tests to determine pulp vitality in various teeth

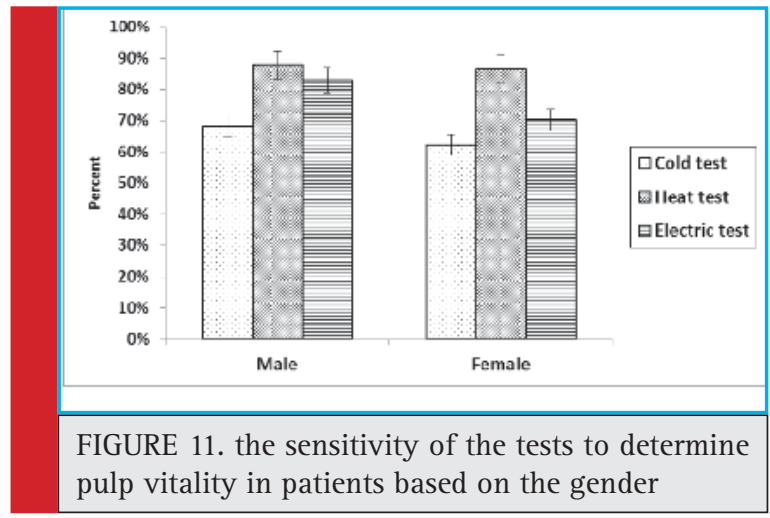

According to the figure 8, primary test (98\%) and cold test (94.1\%) had higher Specificity of the tests for determine pulp vitality in molar teeth

The sensitivity of the tests to determine pulp vitality in various teeth is shown in figure 9. As observed heat test had better sensitivity for anterior, pre-molar and total teeth while electric test had higher sensitivity for molar (figure 9).

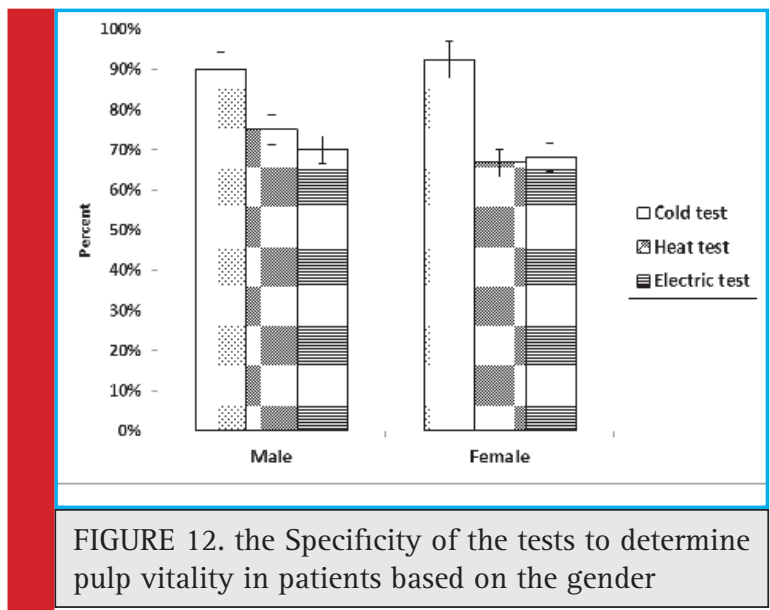



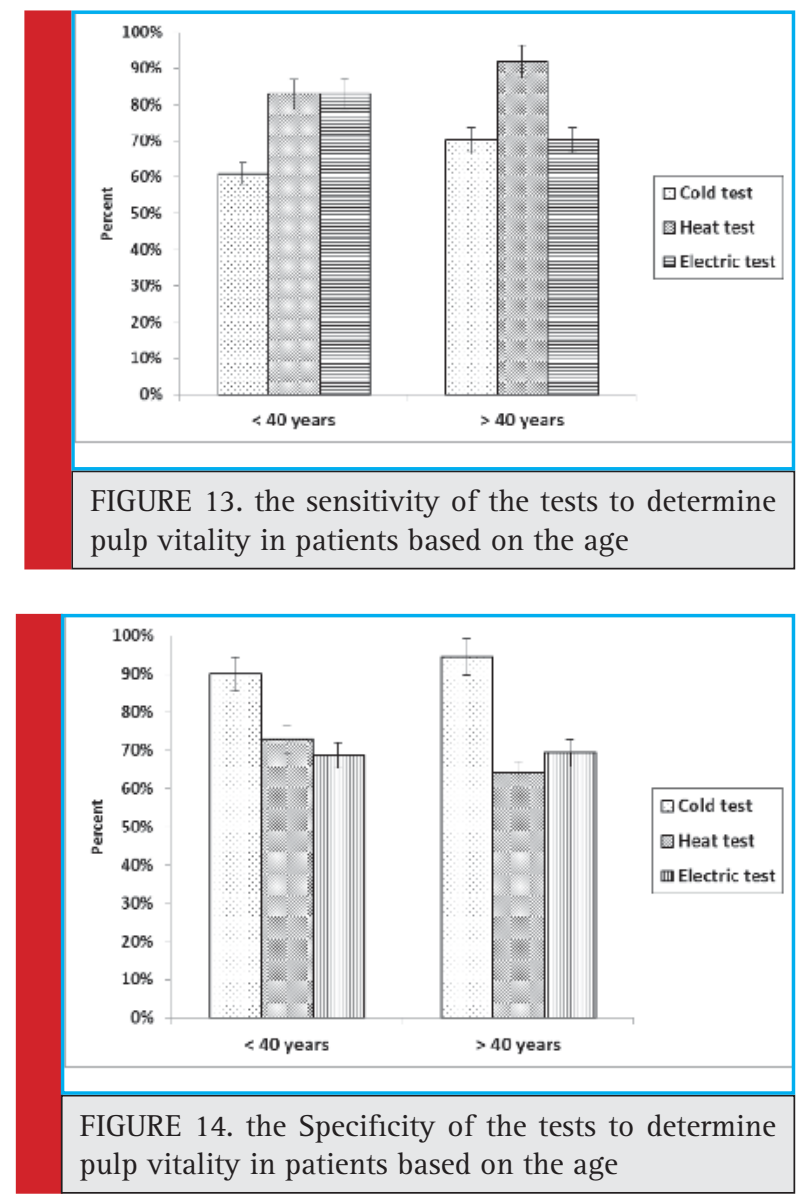

Based on the results of the figure 10, primary test and cold test had highest Specificity to determine pulp vitality in various teeth compared to the other tests.

The heat and electric tests had highest sensitivity to determine pulp vitality in both men and women (figure 11).

Interestingly, the cold test had highest Specificity to determine pulp vitality in both gender (figure 12).

As seen in figure 13, cold and heat tests had similar sensitivity to determine pulp vitality in patients aged $<40$ years old while only heat test had higher sensitivity to determine pulp vitality in patients aged $>40$.

Furthermore, cold test had higher Specificity to determine pulp vitality in patents based on their age (figure 14).

\section{DISCUSSION}

The key to developing a treatment plan for oral rehabilitation is a correct diagnosis, and in endodontic treatment, it is important to identify the status of the pulp tissues. To determine the sensitivity of the pulpal nerves, dentists use thermal and electrical tests (Newton et al. 2009). The ideal test should be easy to use, fast, inexpensive, noninvasive, painless, reproducible, and accurate
(Jafarzadeh and Abbott, 2010). Sensitivity, specificity, and positive and negative predictive values were previously developed to characterize the accuracy of given tests (Dastmalchi et al. 2012).

The sensitivity value indicates the ability of a test to identify teeth that are diseased. The specificity value tests the ability of a procedure to identify teeth without disease (Chen and Abbott, 2011). Therefore, it is important to determine the positive and negative predictive values to identify the probability of a correct diagnosis with thermal and electrical pulp tests (Saeed et al. 2011). As seen in this study the accuracy of heat test in anterior teeth was $82.6 \%$, in premolar teeth was $74.5 \%$ and in molar teeth was $72.7 \%$. The accuracy of electric pulp test in anterior teeth was $71.1 \%$, in premolar teeth was $78.1 \%$ and in molar teeth was71.4\%. The accuracy of cold test in anterior teeth was 78/8\%, in premolar teeth was $81.8 \%$ and in molar teeth was $80.5 \%$. Evaluation of pulp vitality is an important diagnostic aspect of treating traumatized teeth. The methods currently used are thermal and electrical stimulation. However, thermal and electric testing has limitations in providing accurate diagnosis (Goho et al. 1999). Several studies have compared different tests to assess sensitivity, but none of them calculated the positive and negative predictive values (Newton et al. 2009; Setzer et al. 2012).

In this study, anterior teeth the heat test had more accuracy than the other one. Studies have shown that cold testing and electric pulp testing are equally reliable for over $80 \%$ of cases in the diagnosis of vital and nonvital pulps (Weisleder et al. 2009). However, this evidence does not confirm the validity of the test in the assessment of the pulpal condition. This appears currently to be a significant limitation in the diagnosis of diseases of the pulp (Mejàre et al. 2012) attempts to correlate the actual threshold value evoked by electric pulp testing with the condition of pulp remain to be elucidated. Pulp nerve fibers are more resistant to necrosis than vascular tissue, and thermal or electric testing of only pulp neural response may also result in false positive results if only the pulp vasculature is damaged (Goho et al. 1999).

In the current study, premolar and molar teeth the cold test has more accuracy than other tests. It is reported that pulpal sensibility testing with Endo Ice and pulp tests are accurate and reliable methods of determining pulpal vitality (Jespersen et al. 2014).

Patients aged 21-50 exhibited a more accurate response to cold. Sex, tooth type, number of restored surfaces, presence of caries, and recent analgesic use did not significantly alter the results of pulpal sensibility testing in this study. Previous studies have demonstrated no gender related differences in perception threshold (Lin et al. 2007; Jespersen et al. 2014) and our findings was similar to the previous reports. Peterson et al. (1998) 
on the ability of thermal and electrical tests to register pulp vitality reported the sensitivity, specificity, positive predictive value and negative predictive value were calculated for each method. The sensitivity was 0.83 for the cold test, 0.86 for the heat test and 0.72 for the electrical test. The specificity was 0.93 for the cold test, 0.41 for the heat test and 0.93 for the electrical test. The positive predictive value was 0.89 for the cold test, 0.48 for the heat test and 0.88 for the electrical test, and the negative predictive value was 0.90 for the cold test, 0.83 for the heat test and 0.84 for the electrical test. This indicated that the probability of a non-sensitive reaction representing a necrotic pulp was $89 \%$ with the cold test, $48 \%$ with the heat test and $88 \%$ with the electrical test. It also indicated that the probability of a sensitive reaction representing a vital pulp was 90\% with the cold test, 83\% with the heat test and 84\% with the electrical test.

\section{CONCLUSION}

In a diagnostic test (cold, heat, and electrical), it is necessary to know the probability that the test will provide the correct diagnosis. In anterior teeth the heat test has more accuracy than other one. In premolar and molar teeth the cold test has more accuracy than other tests.

\section{REFERENCES}

Bender IB. (2000)Reversible and irreversible painful pulpitides: diagnosis and treatment. Aust Endod Journal 26:10-4.

Chen E, Abbott PV. (2011) Evaluation of accuracy, reliability, and repeatability of five dental pulp tests. J Endod 37:1619-23.

Chen E, Abbott PV. Evaluation of accuracy, reliability, and repeatability of five dental pulp tests .J Endod 2011;37:1619-1623.

Dastmalchi N, Jafarzadeh H, Moradi S.(2012) (Comparison of the efficacy of a custom-made pulse oximeter probe with digital electric pulp tester, cold spray, and rubber cup for assessing pulp vitality. J Endod 38:1182-6.

Emshoff R, Emshoff I, Moschen I, Strobl H. (2004) Diagnostic characteristics of pulpal blood flow levels associated with adverse outcomes of luxated permanent maxillary incisors. Dent Traumatol 20:270-5.

Goho C. (1999)Pulse oximetry evaluation of vitality in primary and immature permanent teeth Pediatr Dent 21:109-113

Gopikrishna V, Pradeep G, VenkaTeshbabu N. (2009) Assessment of pulp vitality: a review. International Journal of Paediatric Dentistry 19: 3-15.

Gopikrishna V, Tinagupta K, Kandaswamy D. (2007) Evaluation of efficacy of a new custom-made pulse oximeter dental probe in comparison with the electrical and thermal tests for assessing pulp vitality. J Endod 33:411-4.

Jafarzadeh H, Abbott PV.(2010) Review of pulp sensibility tests. Part I: general information and thermal tests. Int Endod J 43:738-62.

Lin J, Chandler N, Purton D, Monteith B (2007) Appropriate electrode placement site for electric pulp testing first molar teeth. Journal of Endodontics 33, 1296-8.

Lin J, Chandler NP.(2008) Electric pulp testing: A review. Int Endod Journal; 41:365-74.

Mejàre IA, Axelsson S, Davidson T.(2012) Diagnosis of the condition of the dental pulp: a systematic review. International Endodontic Journal 45, 597-613.

Myers JW. (1998) Demonstration of a possible source of error with an electric pulp tester. J Endod 24:199-200.

Newton CW, Hoen MM, Goodis HE. (2009) Identify and determine the metrics, hierarchy, and predictive value of all the parameters and/or methods used during endodontic diagnosis. J Endod 35:1635-44.

Newton CW, Hoen MM, Goodis HE. (2009) Identify and determine the metrics, hierarchy, and predictive value of all the parameters and/or methods used during endodontic diagnosis. J Endod 35:1635-44

Peterson K, Soderstrom C, Kiani-Anaraki M, Ltvy G.(1999) Evaluation of the ability of thermal and electrical tests to register pulp vitality. Endod Dent Traumatol 15: 127-1 31 .

Rutsatz C, Baumhardt SG, Feldens CA. (2012) Response of pulp sensibility test is strongly influenced by periodontal attachment loss and gingival recession. J Endod 38:580-3.

Saeed MH, Mazhari NA, Al-Rawi NH. (2011) The efficacy of thermal and electrical tests to register pulp vitality. J Int Dent Med Res 4:117-22.

Samuel SS, Thomas AM, Singh N. A (2014) Comparative study of pulse oximetry with the conventional pulp testing methods to assess vitality in immature and mature permanent maxillary incisors Journal of Health and Research 1(4): 235240.

Setzer FC, Kataoka SH, Natrielli F. (2012) Clinical diagnosis of pulp inflammation based on pulp oxygenation rates measured by pulse oximetry. J Endod 38: 880-3.

Villa-Chavez C E., Patino-Marın N, Loyola-Rodrıguez JP, Zavala-Alonso NV, Martınez-Castanon GA, Medina Solıs CE.(2013) Predictive values of thermal and electrical dental pulp tests: A clinical study. J Endod 39:965-969.

Weisleder R, Yamauchi S, Caplan DJ, Trope M, Teixeira FB (2009) The validity of pulp testing: a clinical study. Journal of the American Dental Association 140, 1013-7.

Yu C, Abbott PV. (2007)An overview of the dental pulp: Its functions and responses to injury. Aust Dent J 52:S4-16. 